\title{
Ground State Nucleon, Dark Energy and Dark Matter
}

\author{
Gintautas P. Kamuntavičius \\ Vytautas Magnus University, Kaunas, Lithuania \\ Email: g.kamuntavicius@gmf.vdu.It
}

Received 25 March 2016; accepted 23 April 2016; published 26 April 2016

Copyright (C) 2016 by author and Scientific Research Publishing Inc.

This work is licensed under the Creative Commons Attribution International License (CC BY). http://creativecommons.org/licenses/by/4.0/

CC) (i) Open Access

\begin{abstract}
The recently introduced model of the nucleon as a system of three point particles predicts all characteristics of the proton and neutron with experimental precision only at condition that nucleon is the loosely bound state in system of deep potential wells. The model's Hamiltonian contains ground state with the same parity and spin as the nucleon but with other different characteristics. Existence of this ground state nucleon means that the visible Universe is composed of excited matter. The direct transition between excited and ground states is strictly forbidden, however, stimulated emission can ignite such process. Most likely, corresponding conditions realize at supernova explosion. It is shown that presence of this matter, composed of ground state nucleons, in Universe gives the chance for consistent explanation of dark matter and dark energy phenomena.
\end{abstract}

\section{Keywords}

Solutions of Wave Equations (Bound States), Potential Models, Proton and Neutron, Dark Matter, Dark Energy

\section{Introduction}

The present understanding of fundamental structure of matter is the Standard Model of Elementary Particles (SM), based on assumption that the structure of hadrons is explainable in terms of quark configurations. However, hadrons, excluding only stable proton and neutron, referred as particles, are really extremely short lived resonances, and each of them can be produced over a broad range of excitation energies, and the energies overlap for different resonances. Moreover, the quark model predicts far more resonances than having yet been observed and has a disturbingly large number of parameters whose origins and numerical values are not explained. The SM considers the proton and neutron as the nucleon-the lowest energy state of a family of hadrons that can be constructed from up $(u)$ and down $(d)$ valence quarks. Despite of surprisingly successful SM description of 
resonances, more than a half of century lasting attempts to apply it for proton and neutron are not so impressive.

On the other side, namely the proton and neutron with slightly modified intrinsic structure compose the atomic nuclei, accounting for nearly all the visible mass in the Universe (not less than 0.999), hence the incompletely understood structure of these particles causes problems with atomic nuclei, but especially with description of nuclear matter of such objects as neutron stars and black holes. The outstanding theoretical challenges in nuclear astrophysics, such as collapse of evolved star to structures, which densities are several times larger than nuclear matter densities, the origin of pulsars beams, structure of black holes, the origin of dark matter and dark energy cannot be explained in terms of recent SM.

Therefore, the possibilities of SM modification for visible matter description are already investigated, and one needs more successful model of the nucleon. The article is namely devoted for brief introduction of recently obtained problem solution and presentation of new point of view to structure and evolution of the Universe, following from this innovation.

The paper is organized as follows. In Section 2, we perform the analysis of origins of SM and suggest the modifications of model, necessary for description of the nucleon. In Section 3, we present the main results of the recently introduced model of nucleon as a system of three point particles, which dynamics is governed by Schrödinger equation able to describe all known characteristics of the proton and neutron with experimental precision. Section 4 is devoted for investigation of ground states of introduced Schrödinger's equations, which excited states describe the proton and neutron. In Section 5, we comment different exotic phenomena, caused by ground state proton and ground state neutron presence in the Universe as particles, composing dark matter and being able to predict dark energy phenomenon, accelerating the expansion of the Universe. Finally, we conclude in Section 6.

\section{SM Problems with the Nucleon}

The earliest electron scattering experiments have shown that electric charge and magnetism distributions correspond the point-like electrically charged constituents present in the proton. The proton behaved as if it was made out of point like objects of spin one-half. The further experiments soon indicated that constituents of the proton had a positive parity and the baryon number equal $1 / 3$. These were the first definitive evidences for the quark substructure of the proton and neutron. Moreover, the point-like behavior of quarks at short distance and the strong confining force at large distance, known as asymptotic freedom [1] was the one of most important implications of quark model.

Nearly all of the known hadrons seem to be explainable in terms of the simplest quark configurations with all quarks in ground states, i.e. states, corresponding angular momentum that equal zero. In this approximation, some of measured properties of hadrons can be reproduced surprisingly well but the challenge to the quark model lay in the spin and statistics puzzle. If the baryon $J=1$ octet and $J=3$ decuplet are taken to be composites of three quarks, all in relative $s$-states, then the corresponding wave functions appear to be symmetric in spin-flavor, in conflict with the Pauli exclusion principle. As explicit examples consider the $\Omega^{-}$, formed of three identical strange $(s)$ quarks, sss, or the $\Delta^{++}$, an isospin 3 state made of three up quarks, uuu.

To reconcile the success of the quark model with the requirement that fermion wave functions are antisymmetric, one of the possibilities is to hypothesize that each quark flavor comes in three distinguishable species, which are labeled by the primary colors red, green, and blue. By definition, all baryons are color singlets, which wave functions may then be antisymmetrized in color. Namely this method of wave function antisymmetrization gives not far of experimental value the relation of magnetic momenta of proton and neutron, equal -3/2 [2] (experimental value is -1.459898). This idea soon was realized in form of Quantum chromodynamics (QCD), called as theory of strong interactions among quarks, derived from the color gauge symmetry $S U(3)_{c}$ with eight gluons, defined as carriers of this interaction. However, after more than half of a century of intensive investigations QCD cannot even explain the structure of spin of nucleon [2]-[4], not speaking about the entire set of characteristics of the proton and neutron, including masses, magnetic momenta, charge radii, form factors and so on measured with high precision [5]. The complexity of problem and characteristic results of this kind can be found in recent publications such as [6] [7] and many others.

As it is shown below, the main problem of SM concerning the proton and neutron is namely the definition of nucleon as ground state of hadrons family. The rich set of known and remarkably different characteristics of the proton and neutron requires excited configurations presence in nucleon wave function expansion. 


\section{The Schrödinger's Nucleon}

Let us consider nucleon as an usual quantum system, i.e. system, composed of point-like particles (PP), corresponding recommendations for quark structure of neutron and proton, which dynamics is governed by Schrödinger equation. These particles cannot be called as quarks because widely accepted definition of quarks in SM and QCD is based on assumption that they are particles with intrinsic characteristics such as flavor and color. For the successful description of nucleon only two of them: $u$ and $d$, considered as different structureless (point-like) particles are necessary. The sum of masses of constituents of such system has to be larger than the mass of the entire system. However, for nucleon the difference of these masses, defined usually as binding energy, is senseless due to baryon number conservation, forbidding the split down of the nucleon into composing particles. The Galilei invariance of formalism is crucial; hence elimination of center of mass kinetic energy operator from Hamiltonian is necessary. After this operation the Hamiltonian contains only two Jacobian variables, hence the same dependence has to be in wave functions. All operators of observables have to be rewritten in these variables [8] [9]. Obviously, the potential of point particles interactions contains Coulomb part and some simple potential, keeping the nucleon as bound system, are also necessary. The nucleon does not have bound excited states; hence the potentials of PP interactions have to be of finite depth. The best form of these potentials for translation-invariant Hamiltonian is three-dimensional harmonic-oscillator, together with kinetic energy operator invariant in respect of $S U(3)$ transformations in corresponding space. Three different pairs of PP are possible $(u u, u d$ and $d d$ ), hence one needs at least six parameters (depth and range for every one) for the definition of these potentials. Together with two undefined masses of PP for fitting eight free parameters of nucleon's Hamiltonian appear. However, only six characteristics of the proton and neutron (masses, magnetic momenta and charge radii) are known with high enough precision. Thus, two parameters have to be eliminated from the list. In [9] this problem is solved by defining equal ranges of all three potentials.

More precisely, the potentials have the following form:

$$
V_{\alpha \beta}\left(\left|\mathbf{r}_{j}-\mathbf{r}_{k}\right|\right)=-V_{\alpha \beta}+\frac{1}{2} k_{\alpha \beta}\left(\mathbf{r}_{j}-\mathbf{r}_{k}\right)^{2},
$$

where $\alpha \beta=u u, u d$ and $d d$, and $V_{\alpha \beta}>0$ is depth of a well, while $k_{\alpha \beta}>0$ is the spring constant. The condition for these potentials is that they cannot gain positive values. When $\left(\mathbf{r}_{j}-\mathbf{r}_{k}\right)^{2}=2 V_{\alpha \beta} / k_{\alpha \beta}$, the potential equals zero and remains equal zero at larger distance. As mentioned, this condition is necessary to ensure the absence of bound excited states of nucleon. Namely this relation defines the range of potential, taking the same value for all pairs of PP. Therefore, only depths of these potentials are different.

After this modification the basic Hamiltonian (without Coulomb potential, considered as perturbation) of model has the form, analogous for both, proton and neutron ( $\sigma=1$ and 2 respectively):

$$
H_{\sigma}^{(0)}\left(\xi_{1}, \xi_{2}\right)=H_{\sigma 1}\left(\xi_{1}\right)+H_{\sigma 2}\left(\xi_{2}\right) .
$$

Here

$$
H_{\sigma \rho}\left(\xi_{\rho}\right)= \begin{cases}-\frac{(\hbar c)^{2}}{2 v_{\sigma \rho} c^{2}} \Delta_{(\rho)}+\frac{1}{2} \varkappa_{\sigma \rho}\left(\xi_{\rho}^{2}-\xi_{\sigma \rho}^{2}\right), & \text { if } \xi_{\rho}<\xi_{\sigma \rho} \\ -\frac{(\hbar c)^{2}}{2 v_{\sigma \rho} c^{2}} \Delta_{(\rho)}, & \text { if } \xi_{\rho} \geq \xi_{\sigma \rho}\end{cases}
$$

Reduced masses $v_{\sigma \rho}$ and $\varkappa_{\sigma \rho}, \xi_{\sigma \rho}$, all expressible as functions of six introduced parameters-two PP masses, three depths and one range of potentials of PP interactions, are different for proton and neutron.

The eigenfunction of Hamiltonian (3.3) equals the product of spherical harmonic and radial function. Radial function, corresponding the eigenvalue $E_{n \lambda}^{(\sigma \rho)}$ (here $\lambda$ is angular momentum quantum number, while $n=0,1$, 2 ... equals the number of eigenfunction nodes) is proportional the degenerate hypergeometric function in the area where a potential does not equal zero. In the area where a potential equals zero, radial function is spherical Hankel function of imaginary argument [9].

The eigenfunction of intrinsic Hamiltonian (3.2) equals the product of eigenfunctions of basic Hamiltonians, 
corresponding to different Jacobi coordinates, with bound orbital angular and spin momenta. Angular momentum of nucleon is obtained by binding Jacobi angular momenta and spin momenta of PPs, thus spatial and spin part of the wave function has the expression:

$$
\left|\left(\left(\lambda_{1}, s_{1}\right) j_{1},\left(\lambda_{2},\left(s_{2}, s_{3}\right) s_{23}\right) j_{23}\right) j=1 / 2, m\right\rangle .
$$

Here the parentheses indicate the operation of momenta binding; $\lambda_{1}$ is angular momentum of the first Jacobi coordinate $\xi_{1} ; s_{1}=1 / 2$ is spin momentum of the first PP; $\lambda_{2}$ indicates the angular momentum of these cond Jacobi coordinate $\xi_{2}$, for which, according to Jacobi tree structure [8], the second and third PPs spinmomenta $s_{2}$ $=1 / 2$ and $s_{3}=1 / 2$ need to be set. The $j_{1}, j_{23}$ are total momenta of respective Jacobian subsystems. Their sum $j=1 / 2$ equals the nucleon momentum, i.e. its spin.

Obvious restrictions for quantum numbers, caused by nucleon characteristics, appear. Firstly, the total momentum of nucleon equals $1 / 2$, this has already been expressed in the wave-function. Secondly, orbital parity of a nucleon is positive, therefore $\lambda_{1}+\lambda_{2}$ has to be an even number. Finally, the second and third particles are identical; therefore, the corresponding part of the wave-function needs to be antisymmetric with regard to spin and orbital degrees of freedom permutations. So, $\lambda_{2}+s_{23}$ also has to be an even number.

These wave functions were applied for different characteristics of nucleon calculation and expression in terms of parameters of Hamiltonian. The fitting has shown that for the neutron and proton description applying the eigenfunctions of corresponding Hamiltonians necessary is superposition of two excited configurations with Jacobi angular momenta $\lambda_{1}=\lambda_{2}=1$ and nodeless radial functions:

$$
\frac{1}{\sqrt{2}}\left[\left|\left(\left(1, \frac{1}{2}\right) \frac{1}{2},\left(1,\left(\frac{1}{2}, \frac{1}{2}\right) 1\right) 1\right) \frac{1}{2}, m\right\rangle+\left|\left(\left(1, \frac{1}{2}\right) \frac{3}{2},\left(1,\left(\frac{1}{2}, \frac{1}{2}\right) 1\right) 2\right) \frac{1}{2}, m\right\rangle\right] .
$$

The set of parameters of model, predicting the known data of nucleon with experimental precision, is published in [9]. The other characteristics of neutron and proton, such as elastic form factors, different rms radii and Zemach momenta, are present in [10] and [11]. All obtained results do not contradict the known information about the structure of the proton and neutron.

\section{Ground State Nucleon}

Therefore, successful model of the proton $(p)$ and the neutron $(n)$ predicts they are loosely bound states in system of deep potential wells. As mentioned, the intrinsic Hamiltonians of model are free of bound excited states of $\mathrm{p}$ as well as $\mathrm{n}$; however, they have ground states of this kind. As it will be shown later, namely presence of these states, ground state proton $\bar{p}$ and ground state neutron $\bar{n}$ can explain obvious but not completely clear connection between nuclear properties and the nature of the Universe and give interesting information about new sort of matter and some phenomena, which origin is not yet understood applying traditional approaches. For the sake of simplicity let us call these particles as dark proton and dark neutron.

The angular and spin part of the wave function of dark nucleon has the same structure as the wave function of nucleon, present in Equation (3.4) with both angular momenta $\lambda_{1}=\lambda_{2}=0$, i.e.:

$$
\left|\left(\left(0, \frac{1}{2}\right) \frac{1}{2},\left(0,\left(\frac{1}{2}, \frac{1}{2}\right) 0\right) 0\right) j=\frac{1}{2}, m\right\rangle .
$$

The corresponding radial wave functions again are nodeless, like wave functions of ordinary nucleon.

The energies and other main characteristics of these particles are present in Table 1.

Masses of $\bar{p}$ and $\bar{n}$ are the most interesting result. They are negative due to character of potential wells, necessary to describe the proton and neutron. Moreover, absolute values of these masses coincide with good precision with masses of proton and neutron. However, these particles cannot be identified as Dirack's antiparticles because they correspond the solutions of the same Schrödinger equation with absent mechanism of charge inversion.

As it will be shown later, the best consideration of obtained results is as follows. Let us define the potentials of PP interaction, following from nucleon parameters fitting, as a short range gravitation. Really, the putative graviton has not yet been found, therefore this is only choice for point-like particles interaction, not stranger in 
Table 1. Main characteristics of dark proton and dark neutron.

\begin{tabular}{ccc}
\hline & $\bar{p}$ & $\bar{n}$ \\
Mass $(\mathrm{MeV})$ & -938.2720454 & -939.5653813 \\
Magnetic momentum $\left(\mu_{N}\right)$ & -0.9248409550 & 1.946549588 \\
Charge radius $(\mathrm{fm})$ & 0.4036284062 & \\
Mean-square charge radius $\left(\mathrm{fm}^{2}\right)$ & & -0.02474114545 \\
Mass radius $(\mathrm{fm})$ & 0.3584056883 & 0.3570377735 \\
\hline
\end{tabular}

comparison with long range gravitation, tractable as space curvature. If so, the next conclusion is obvious: one has to define the mass, corresponding negative energy of dark particles, as gravitational mass according to definition

$$
m_{g}=E / c^{2}
$$

Let the interaction of masses is defined by well-known formula with gravitational constant, modified only by signs of interacting masses, resulting in repulsion of masses, composed of ordinary and dark nucleons.

For ordinary nucleons the gravitational mass is positive and with high precision equals the inertial mass. Therefore, the inertial mass, by definition taking only positive values, has to be defined as

$$
m_{i}=\left|m_{g}\right| \text {. }
$$

Let us study now, what interesting conclusions follow from this definition.

\section{Evolution of a Star Remnant}

Obviously, both new particles are very compact and $\bar{n}$ is the most stable system, composed of three PPs. Once created, the dark proton after a weak process

$$
\bar{p}=\bar{n}+\mathrm{e}^{+}+v
$$

appears as dark neutron $\bar{n}$.

Therefore, dark neutron is the best candidate for particles, composing the extremely dense matter, invisible in usual regions of electromagnetic radiation. Due to compactness of these particles (mass radius of neutron equals $0.768 \mathrm{fm}$ [10], while mass radius of $\bar{n}$ is more than two times smaller and equals $0.357 \mathrm{fm}$ ) such matter can reach densities, by order larger than nuclear matter density.

The interaction of these particles is very weak, governed only by gravitation. The electromagnetic interaction is absent due to neutrality of $\bar{n}$. The strong interaction, which carriers are mainly mesons, it is quark and antiquark pairs, cannot play significant role due to extreme binding of PPs, composing the dark nucleon. For ordinary nucleons this interaction is really strong due to loosely bound PPs; therefore, our model predicts that dark neutrons are extremely stable and weakly interacting particles.

The main question is this: How do these particles appear in the Universe?

The reactions, responsible for creation of dark nucleons, are

$$
\left\{\begin{array}{l}
n \rightarrow \bar{n}+\gamma(1.879 \mathrm{GeV}) \\
p \rightarrow \bar{p}+\gamma(1.877 \mathrm{GeV})
\end{array}\right.
$$

The following weak process (5.1) converts dark protons to dark neutrons. The proton and the corresponding dark proton, exactly as neutron and dark neutron, are eigenstates of the same Hamiltonian, thus the interaction with electromagnetic field can cause transitions between these states. The problem is that any transitions of this kind are strictly forbidden. Electric dipole transition operator expectation value equals zero due to positive parity of both wave functions, (3.5) and (4.1). Magnetic dipole transition operator, written in intrinsic variables [9], expectation value vanishes due to orthogonality of both Jacobi subsystems of mentioned wave functions. The operators of higher multiplicity expectation values equal zero due to Wigner-Eckart theorem because total mo- 
menta of both wave functions are equal $1 / 2$.

Only possibility for this transition to happen is finite lifetime of nucleon in respect of such transformations and stimulated emission, developing at some conditions. These conditions realize, when massive star-supernova progenitor-exhausts the nuclear fuel and gravitation starts iron core conversion to easier structures, such as alpha particles and neutrons. Density of core grows and onion-skin structure of progressively lighter elements from the inside out can serve as a mirror for stimulated emission of gamma quanta and produce in center of remnant the clamp of negative gravitational mass. The energy, generated during this process, is enough for observable extremely fast motion outside the outer shells of progenitor, composed of visible matter and at the same time the collapse of core due to gravitational attraction of dark nucleons. This scenario is best possible for the core-collapse supernova mechanism explanation because "...the current status of the theory for the mechanism and the systematics of core-collapse explosions is ambiguous, if not confusing" [12].

Further development of remnant is dependent mainly on progenitor mass. If this mass is large enough for all core conversion to system, composed of negative gravitational mass, and happens the event, called as black hole creation after hypernova explosion. Therefore, the massive progenitors generate massive cores, composed of dark neutrons. At smaller mass of progenitor core, created after supernova explosion, together with negative gravitational mass contains also some amount of ordinary mass. Due to angular momentum conservation such remnant, called as neutron star, fastens rotation and takes prolate form like disc or more complex, not axially symmetric, shape. Namely along these largest dimensions the best conditions for stimulated emission appear. The crystalline crust of neutron star can serve as semitransparent mirror and the process like the one in laser starts. This scenario gives a perfect explanation of observable orientation of rotation and emission axes of pulsars (for axially symmetric pulsar the emission axis is perpendicular to the rotation axis) and coherence of corresponding radiation. Young pulsars with significant amounts of ordinary matter produce powerful beam of $\gamma$-quanta with defined above energies. Later on, when the intensity of beam reduces, the crystalline crust of pulsar can redshift the penetrating radiation, converting it for old pulsars to radio waves.

The further evolution of pulsar can develop in a following way. The born dark neutrons accumulate in central region of ordinary matter disc. This region is stable in respect of negative mass outflow to space until the amount of negative mass is small enough. Growing amounts of negative gravitational mass in these regions can cause different phenomena, happening during star remnant evolution. These are the powerful shock waves that travel outward through the mantle of the star remnant and ejections of visible matter to space after negative mass amount riches critical value.

Therefore, after supernova explosion, excluding complete disassembling of remnant at minimal possible mass of progenitor, sooner or later the core appears as structure, composed of dark neutrons. The mass of these structures can grow due to attraction and collapse of remnants with negative gravitational mass. This scenario can explain masses of merging black holes, producing recently observed gravitational waves [13], about twice as massive as they should be according to the current theories of how black hole form a star. For the first time in history, scientists have physical proof that pairs of black holes will sometimes collide and mush together to form a single, bigger black hole. Moreover, all of the observed black hole systems are found in low stellar density galactic fields and dynamically formed merging binary black holes are ejected from the host cluster before merger. These observations are in full accordance with our explanation, stating that no ordinary, i.e. positive gravitational masses can be present in closest vicinity of these black holes due to gravitational repulsion.

\section{Dark Energy and Dark Matter}

Finally, when star remnant produces big enough amount of dark neutrons, the observable matter and matter with negative gravitational mass will separate. The ordinary matter spreads out in galaxy forming clouds of gas and dust for following generation of stars. The clumps of negative mass due to gravitational repulsion move out of galactic discs to extragalactic space. Namely this scenario can explain the stability of galactic discs and not growing large galaxy mass due to the absorption of dwarf galaxies. From the other side, the appearance of these clumps before escape from disc interacts with visible matter, present in the disc; hence regions of galaxy with older stars are spread in thicker layer and have larger velocity spreads than younger stars do. This distribution of stars (thick disk) cannot be explained as result of ordinary gravitational attraction, see [14]. Obviously, these clumps move to extragalactic space, compacting nearby dwarf galaxies and preventing them from tidal destruction due to big galaxy gravitation. The attraction of these masses creates bigger and bigger structures of this kind 
and presence of these objects in space causes expansion of the Universe. Due to the absence of mechanism for disappearance of this matter, the growing amount of negative gravitational mass in the Universe has caused the originally slowing expansion during the first half of the Universe life to reaccelerate [15]. The accelerated expansion of the Universe has been the biggest recent challenge, not consistent with dark matter phenomenon and not explainable yet in another way than given above. The best characteristic of existing situation is given in [16]: "the label "dark energy" encapsulates our helplessness".

Concerning the development of Galactic Center supermassive black hole; according to our suggestion, it is composed of dark neutrons and looks like the black hole, defined according to a standard consideration. This object of negative gravitational mass, situated in the center of galactic bulge, absorbs clumps of negative mass matter, produced by pulsars. This structure grows until mass reaches value, destroying the stability of Galactic center. The final stage of such scenario is escape of black hole from bulge, creating ultrafast outflows of remnants of visible matter, observable as active galactic nuclei and bright regions around its central black hole identified as quasars.

Moreover, our model can explain dark matter consistence with dark energy. According to the traditional approach, for the explanation of measured galactic rotation curves, significantly bigger mass of galaxy is necessary. For our model the key to explain this phenomenon is the evaporation of matter from galaxy disc to halo and later on to extragalactic space. Strange character of visible matter movement in halo can be caused by repulsion of visible matter and present in halo clumps of dark matter, which gravitational mass is negative. On the other side, the infrared observations showed that stellar mass assembly favored more massive systems at earlier epochs [17]. Bright galaxies of the early universe, built from clouds, composed mainly of protons and alphas, were big structures with many gigantic stars, significantly more massive as now. Evolution of these stars resulted in big amounts of negative mass appearance, hence evaporation and lowering the mass and moment of inertia of galaxy. This effect due to angular momentum of galaxy conservation causes observable movement of halo stars faster than following from Kepler's laws. Other effect, explanation of which needs bigger than visible masses is stability of galactic clusters. Again, this can be explained as result of gravitational repulsion of negative masses, moving in outer regions of cluster. Finally, one of first observations, which explanation required that the total mass of the Milky Way and Andromeda galaxies had to be much larger than their stellar mass, is character of movement of these galaxies, currently approaching each other. From our point of view, this movement can be caused by nearly parallel discs of both galaxies, hence the streams of clumps of negative gravitational mass move in the same directions, perpendicular to the galaxies discs and cannot resist this process. By a way, some merging big galaxies, present in Hubble gallery, have parallel discs.

\section{Conclusions}

Therefore, a successful model of nucleon has shown that proton and neutron are the systems of point particles, occupying excited states of three-particle Schrodinger equation. The basic of our approach is taken into account Galilei invariance of nucleon Hamiltonian and wave functions. This invariance, fundamental for relativity theory, until the last time due to problems with identical particles wave functions antisymmetrisation and problems with different operators of observables expansion in Jacobi variables, was ignored in quantum mechanics. The second basic feature of our approach is introduction of potentials of PP interaction, equal three dimensional harmonic oscillator ones, so that Hamiltonians dependent on different Jacobi variables, are symmetrical in respect of $S U$ (3) transformations, characteristic for Standard Model dynamics. This symmetry is significant at conditions, characteristic for resonances because at high excitations, i.e. high kinetic energies, the fine distinctions of particle masses and interaction potentials do not play a significant role. The character of applied potentials shows some links between theory of resonances-Standard Model and present model of bound states of hadrons. The third basic feature of our approach is finite depth of all potential wells, necessary to ensure absence of excited bound states of our Hamiltonian because the only stable hadrons are the proton and neutron. As a result, this approach eliminates necessity to mark quarks as colored objects because if excited orbital states are allowed for nucleon, they can also be present in expansions of the wave functions of mentioned resonances, composed of identical quarks, such as $\Delta^{++}$and $\Omega^{-}$.

The excited bound states of our Hamiltonians for the proton and neutron are absent but ground states with quantum numbers (parity and spin), coinciding with ones for proton and neutron are present. Jacobian orbital angular momenta equals zeroes, hence these states are the best for nucleon of SM identification. However, other 
characteristics of these states do not correspond to any known particle. Moreover, due to deep potential wells, the energies of these states are negative and absolute values of ones are equal the masses of ordinary neutron and proton. The best consideration of this result is to define this energy as a corresponding the negative gravitational mass of these particles. The inertial mass, in complete correspondence with known relation of these masses for ordinary particles, has to be defined as equal the absolute value of gravitational mass.

To some extent, the reactions (5.2) look like annihilation of particle and antiparticle. The nucleon disappears, and the born dark nucleon moves out due to repulsion and the only observable result is gamma quantum of defined energy. The difference of these acts is as follows: at annihilation of particle and antiparticle due to momentum conservation, necessary are two quanta, every having energy, equal to the mass of annihilating particles. The best known process of this kind is annihilation of electron and positron, producing quanta with energy, equal to the mass of electron. Our process creates only one gamma quantum of double energy. Several groups have found strong evidence for extended emission of the gamma rays from the Galactic Center using data from the Large Area Telescope aboard the Fermi Gamma-ray Space Telescope and have found an excess in $\gamma$ rays over known astrophysical backgrounds which peak at energy, equal approximately $2 \mathrm{GeV}$ [18]. One possible source of this radiation is a previously unidentified population of millisecond pulsars in the Galactic Center bulge [19] [20].

Therefore, from our consideration it follows that exotic objects, such as big stars remnants and dark matter, are composed mainly of dark neutrons and have negative gravitational mass. Gravitation is the main force acting between these masses. They are weakly interacting elements of matter, able to compose larger structures of significantly larger than nuclear matter densities. It looks like they finally accumulate in voids-big cavities of the Universe, having no visible matter. Most probably, the gravitational interaction of these absolutely stable particles converts these clumps into structures such as ordinary protostars, i.e. with permanently growing kinetic energy and temperature. In ordinary star, the thermonuclear synthesis starts with deuteron production due to gain in energy converting one proton to neutron because the difference of masses of proton and neutron, equal 1.3 $\mathrm{MeV}$, is less than binding energy of deuteron, equal $2.2 \mathrm{MeV}$. No reactions like this can start in clump of negative gravitational mass due to mentioned above extreme binding of PPs, composing $\bar{n}$, hence the process of negative gravitational mass deuteron, i.e. the system, composed of $\bar{n}$ and $\bar{p}$, and more complex systems creation cannot stop this process. After some time, when temperature reaches necessary value, the energy of this gigantic clump of negative mass can be enough to excite and convert this system to ordinary neutrons and protons and start process like Big Bang without any problems with antimatter annihilation.

One more conclusion, following from present consideration, is that reaction (5.1) can explain the excess of positrons in the Universe, necessary for intensive annihilation with electrons, producing the photons with characteristic well defined energy, equal $511 \mathrm{keV}$. Almost 40 years after discovery, the origin of this phenomenon remains unknown and makes this problem one of the most intriguing ones in high energy astrophysics [21]. This emission is strongly concentrated toward the Galactic bulge, and hence follows directly from our model of negative gravitational mass appearance. Moreover, the distribution of emitted positrons kinetic energies present in [21], do not exceed 1.3 MeV in complete correspondence with our prediction. By the way, this reaction also explains the origin of intensive jets of neutrinos after the supernova explosion.

Finally, our model explains numerous unsuccessful efforts to observe dark particles in laboratories on Earth [22]. These particles are absent here due to gravitational repulsion with ordinary matter, composing all observable world. Moreover, for obvious reasons, they cannot be produced in colliders. On the other side, one has indirect evidences for these particles existence-accelerating expansion of the Universe and reduction of star formation rate in our Galaxy [23], due to mass evaporation to extragalactic space.

Concluding, present results are the best illustration of unity of the Universe. The successful model of the smallest quantum system-nucleon, taking into account cosmological principle, i.e. invariance of the Universe in respect of spatial translations and rotations, creates unique possibility to understand the evolution of the biggest structures-stars, galaxies and finally-evolution of entire Universe.

\section{References}

[1] Gross, D.J. (2005) Nobellecture: The Discovery of as Symptotic Freedom and the Emergence of QCD. Reviews of Modern Physics, 77, 837-849. http://dx.doi.org/10.1103/RevModPhys.77.837

[2] Thomas, A.W. and Weise, W. (2001) The Structure of the Nucleon. Wiley-VCH, Berlin. 
http://dx.doi.org/10.1002/352760314X

[3] Bernard, V., Epelbaum, E., Krebs, H. and Meißner, U.-G. (2013) New Insights into the Spin Structure of the Nucleon. Physical Review D, 87, Article ID: 054032. http://dx.doi.org/10.1103/PhysRevD.87.054032

[4] Aidala, C.A., Bass, S.D., Hasch, D. and Mallot, G.K. (2013) The Spin Structure of the Nucleon. Reviews of Modern Physics, 85, 655-691. http://dx.doi.org/10.1103/RevModPhys.85.655

[5] Olive, K.A., et al. (Particle Data Group) (2014) Review of Particle Physics. Chinese Physics C, 38, Article ID: 090001. http://dx.doi.org/10.1088/1674-1137/38/9/090001

[6] Capitani, S., Morte, M.D., Djukanovic, D., von Hippel, G., Hua, J., Jäger, B., Knippschild, B., Meyer, H.B., Rae, T.D. and Wittig, H. (2015) Nucleon Electromagnetic Form Factors in Two-Flaw or QCD. Physical Review D, 92, Article ID: 054511. http://dx.doi.org/10.1103/PhysRevD.92.054511

[7] Green, J., Meinel, S., Engelhardt, M., Krieg, S., Laeuchli, J., Negele, J., Orginos, K., Pochinsky, A. and Syritsyn, S. (2015) High-Precision Calculation of the Strangenucleonelectro Magnetic Form factors. Physical Review D, 92, Article ID: 031501. http://dx.doi.org/10.1103/PhysRevD.92.031501

[8] Kamuntavičius, G.P. (2014) Galilei Invariant Technique for Quantum System Description. Journal of Mathematical Physics, 55, Article ID: 042103. http://dx.doi.org/10.1063/1.4870617

[9] Kamuntavičius, G.P. (2014) Nucleon as a Nonrelativistic Three Point Particles System. SOP Transactions on Theoretical Physics, 1, 44-56. http://dx.doi.org/10.15764/TPHY.2014.04004

[10] Kamuntavičius, G.P. (2015) Structure of Schrödinger's Nucleon: Elastic Form-Factor Sand Radii. Journal of Applied Mathematics and Physics, 3, 1352-1360. http://dx.doi.org/10.4236/jamp.2015.310162

[11] Kamuntavičius, G.P. (2015) Structure of Schrödinger's Nucleon: Density Distributions and Zemach Momenta. Journal of Applied Mathematics and Physics, 3, 1412-1421. http://dx.doi.org/10.4236/jamp.2015.311169

[12] Burrows, A. (2013) Colloquium: Perspectives on Core-Collapse Supernova Theory. Reviews of Modern Physics, 85, 245-261. http://dx.doi.org/10.1103/RevModPhys.85.245

[13] Abbott, B.P., et al. (2016) Astrophysical Implications of the Binary Black Hole Merger GW150914. The Astrophysical Journal Letters, 818, L22. http://dx.doi.org/10.3847/2041-8205/818/2/122

[14] Sellwood, J.A. (2014) Secular Evolution in Disc Galaxies. Reviews of Modern Physics, 86, 1-46. http://dx.doi.org/10.1103/RevModPhys.86.1

[15] Perlmutter, S. (2012) Nobel Lecture: Measuring the Acceleration of the Cosmic Expansion Using Supernovae. Reviews of Modern Physics, 84, 1127-1149. http://dx.doi.org/10.1103/RevModPhys.84.1127

[16] Frenk, C.S. and White, S.D.M. (2012) Dark Matter and Cosmic Structure. Annalen der Physik, 524, 507-534. http://dx.doi.org/10.1002/andp.201200212

[17] Silk, J. and Mamon, G.A. (2012) The Current Status of Galaxy Formation. Research in Astronomy and Astrophysics, 12, 917-946. http://dx.doi.org/10.1088/1674-4527/12/8/004

[18] Calore, F., Cholis, I., McCabe, Ch. and Weniger, Ch. (2015) A Tale of Tails: Dark Matter Interpretations of the Fermi GeV Excess in Light of Background Model Systematics. Physical Review D, 91, Article ID: 063003. http://dx.doi.org/10.1103/PhysRevD.91.063003

[19] Bartels, R., Krishnamurthy, S. and Weniger, Ch. (2016) Strong Support for the Millisecond Pulsar Origin of the Galactic Center GeV Excess. Physical Review Letters, 116, Article ID: 051102. http://dx.doi.org/10.1103/PhysRevLett.116.051102

[20] Lee, S.K., Lisanti, M., Safdi, B.R., Slatyer, T.R. and Xue, W. (2016) Evidence for Unresolved $\gamma$-Ray Point Sources in the Inner Galaxy. Physical Review Letters, 116, Article ID: 051103. http://dx.doi.org/10.1103/PhysRevLett.116.051103

[21] Prantzos, N., et al. (2011) The $511 \mathrm{keV}$ Emission from Positron Annihilation in the Galaxy. Reviews of Modern Physics, 83, 1001-1056. http://dx.doi.org/10.1103/RevModPhys.83.1001

[22] Undagoitia, T.M. and Rauch, L. (2016) Dark Matter Direct-Detection Experiments. Journal of Physics G: Nuclear and Particle Physics, 43, Article ID: 013001. http://dx.doi.org/10.1088/0954-3899/43/1/013001

[23] Haywood, M., et al. (2016) When the Milky Way Turned-Off the Lights: APOGEE Provides Evidence of Star Formation "Quenching" in Our Galaxy. Astronomy and Astrophysics. arXiv: 0601.03042 [astro-ph.GA]. 